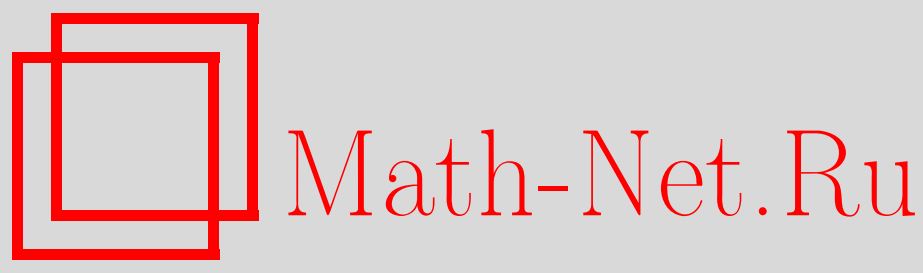

А. В. Киселев, Приближенные формулы для умеренно малых эйкональных амплитуд, ТМФ, 2016, том 188, номер 2, 273-287

DOI: https://doi.org/10.4213/tmf9093

Использование Общероссийского математического портала Math-Net.Ru подразумевает, что вы прочитали и согласны с пользовательским соглашением http://www.mathnet.ru/rus/agreement

Параметры загрузки:

IP : 54.81 .137 .203

26 апреля 2023 г., 13:44:51

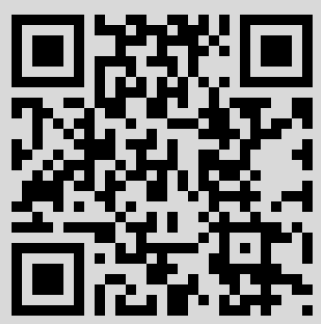




\title{
ФИЗИКА
}

Том 188, № 2

август, 2016

(C) 2016 г.

\author{
А. В. Киселев*
}

\section{ПРИБЛИЖЕННЫЕ ФОРМУЛЫ ДЛЯ УМЕРЕННО МАЛЫХ ЭЙКОНАЛЬНЫХ АМПЛИТУД}

Рассмотрено эйкональное приближение для умеренно малых амплитуд рассеяния. Для того чтобы найти численные оценки этих приближений, выведены формулы, не содержащие функций Бесселя и, как следствие, быстро осциллирующих подынтегральных выражений. Для получения этих формул изучены несобственные интегралы первого рода, содержащие произведения функций Бесселя $J_{0}(z)$. Обобщено выражение с четырьмя функциями $J_{0}(z)$. Также найдены выражения для интегралов, содержащих произведения пяти и шести функций Бесселя. Известное выражение для несобственного интеграла с двумя функциями $J_{\nu}(a z)$ обобщено на случай нецелых значений $\nu$ и комплексных значений $a$.

Ключевые слова: амплитуда рассеяния, эйкональное приближение, несобственные интегралы, функции Бесселя.

DOI: $10.4213 / \operatorname{tmf} 9093$

\section{1. ВВЕДЕНИЕ}

Эйкональное приближение возникло при изучении лучевой оптики, в которой предполагается, что свет распространяется прямолинейно. Это предположение прекрасно работает в случае, когда размер объекта $a$ велик по сравнению с длиной волны света $\lambda$.

В квантовой механике эйкональное приближение хорошо работает для процессов рассеяния частиц с большими начальными импульсами $k$ и малыми углами рассеяния $\theta$. В потенциальном рассеянии эйкональное приближение можно использовать, если выполнено условие

$$
E \gg V(\vec{r}),
$$

где $E$ есть энергия частицы, а $V(\vec{r})$ - потенциал взаимодействия [1], [2]. Сечение рассеяния определяется через амплитуду рассеяния как

$$
\frac{d \sigma}{d \Omega}=\left|A\left(\vec{k}, \vec{k}^{\prime}\right)\right|^{2}
$$

* Институт физики высоких энергий, НИЦ "Курчатовский институт", Протвино, Московская обл., Россия. E-mail: alexandre.kisselev@ihep.ru 
где $\vec{k}$ и $\vec{k}^{\prime}$ суть соответственно начальный и конечный 3 -импульсы частицы. Для дальнейшего рассмотрения удобно определить квадрат переданного импульса:

$$
t=-\left(\vec{k}-\vec{k}^{\prime}\right)^{2}=-2 k^{2}(1-\cos \theta) .
$$

Борновская амплитуда выглядит следующим образом:

$$
A_{\mathrm{B}}\left(\vec{k}, \vec{k}^{\prime}\right)=-\frac{1}{4 \pi} \int d \vec{r} e^{\left(\vec{k}-\vec{k}^{\prime}\right) \vec{r}} V(\vec{r}) .
$$

В эйкональном приближении для сферически-симметричного потенциала амплитуду можно представить в виде (см., например, книгу [3])

$$
A\left(\vec{k}, \vec{k}^{\prime}\right)=i k \int_{0}^{\infty} d b b J_{0}(b \sqrt{-t})\left\{1-e^{i \chi(b)}\right\}
$$

с эйконалом $\chi(b)$, определенным формулой

$$
\chi(b)=-\frac{k}{E} \int_{b}^{\infty} \frac{r d r}{\sqrt{r^{2}-b^{2}}} V(r) .
$$

Заметим, что данный эйконал является преобразованием Фурье борновской амплитуды:

$$
\chi(b)=\frac{1}{2 \pi k} \int d^{2} k e^{-i \vec{k} \vec{b}} A_{\mathrm{B}}\left(\vec{k}, \vec{k}^{\prime}\right)=\frac{1}{k} \int_{0}^{\infty} d t J_{0}(b \sqrt{-t}) A_{\mathrm{B}}(s, t),
$$

где $s=k^{2}$.

В квантовой теории возмущений экспоненцирование амплитуд высокоэнергетических процессов, подобное тому, которое имеет место в глауберовском приближении, было изучено в работе [4]. В статье [5] эйкональное представление выведено в рамках квазипотенциального подхода для малых углов рассеяния и гладких квазипотенциалов [6]. В работе [7] рассмотрено обобщение эйконального подхода, которое автоматически учитывает условие унитарности вне массовой поверхности.

Дифференциальное сечение рассеяния дается выражением

$$
\frac{d \sigma(s, t)}{d t}=\frac{1}{16 \pi s^{2}}|A(s, t)|^{2},
$$

где $A(s, t)$ есть (безразмерная) амплитуда рассеяния, $s$ и $t=-q_{\perp}^{2}-$ переменные Мандельстама. В эйкональном приближении амплитуда определяется формулой

$$
A(s, t)=4 \pi i s \int_{0}^{\infty} d b b J_{0}(b \sqrt{-t})\left\{1-e^{i \chi(s, b)}\right\}
$$

где $b$ - прицельный параметр. В свою очередь эйконал $\chi(s, b)$ в $(2)$ связан с борновской амплитудой $A_{\mathrm{B}}(s, t)$ через преобразование Фурье-Бесселя:

$$
\chi(s, b)=\frac{1}{4 \pi s} \int_{0}^{\infty} d q_{\perp} q_{\perp} J_{0}\left(q_{\perp} b\right) A_{\mathrm{B}}\left(s,-q_{\perp}^{2}\right) .
$$

Если $A_{\mathrm{B}}$ аппроксимируется реджеонами, мы приходим к так называемому реджеэйкональному подходу ${ }^{1)}$.

1) В случае дополнительных пространственных измерений борновская амплитуда получает также вклад от “грави-реджеонов” (реджезованных гравитонов Калуцы-Клейна в $t$-канале) [8]. 
Предположим, что нам требуется численно оценить амплитуду (и соответственно дифференциальное сечение) в кинематической области

$$
s \gg-t \gg m_{\mathrm{N}}^{2}
$$

как, например, это имеет место при изучении взаимодействия высокоэнергетических космических частиц с нуклонами атмосферы. Как можно видеть из уравнений $(2),(3)$, амплитуда определяется через $A_{\mathrm{B}}$ несобственным повторным интегралом, который содержит быстро осииллирующие функции Бесселя. Попытки численно оценить такой интеграл с большой точностью в кинематической области (4) могут натолкнуться на вычислительные трудности.

Сложности в оценке интегралов, содержащих произведения функций Бесселя, были рассмотрены в работах [9], [10]. В статье [9] были изучены интегралы вида

$$
\int_{0}^{\infty} d x f(x) J_{\mu}(a x) J_{\nu}(b x)
$$

где $\mu, \nu$ - неотрицательные целые, а $a, b$ - положительные целые числа. В работе [10] рассмотрено вычисление интегралов, содержащих произведение произвольного числа функций Бесселя:

$$
\int_{0}^{\infty} d x e^{-c x} x^{m} \prod_{i=1}^{k} J_{\nu_{i}}\left(a_{i} x\right) .
$$

Здесь $\sum_{i} \nu_{i}+m>-1$, а $c$ и все числа $a_{i}$ строго положительны. Частные случаи интеграла (5) встречаются в астрофизике [11] и ядерной физике [12].

Как отмечено выше, интегралы, содержащие произведение функций Бесселя, трудно поддаются численным оценкам из-за слабой сходимости и осциллирующего характера данных интегралов. Мы столкнулись с такой проблемой [13], когда потребовалось оценить амплитуду рассеяния космических нейтрино ультравысоких энергий на нуклонах. Энергия космических нейтрино $E_{\nu}$ может достигать $10^{12}$ ГэВ. Соответственно, переданный импульс в формулах, аналогичных уравнениям (1), (2), может меняться вплоть до $\sqrt{-t} \simeq 10^{6}$ ГэВ.

Проблема упрощается, если $|\chi(s, b)| \ll 1$ для всех рассматриваемых $b$ при фиксированных $s$. Тогда можно использовать разложение

$$
1-e^{i \chi}=-i \chi+\mathrm{O}\left(\chi^{2}\right)
$$

и получить хорошо известную формулу $A(s, t) \simeq A_{\mathrm{B}}(s, t)$ (см. приведенное ниже уравнение (12)). Однако может случиться так, что эйконал $|\chi(s, b)|$ умеренно мал (т. е. $|\chi(s, b)|<1$, но $\chi(s, b)=\mathrm{O}(1))$. В этом случае применения разложения (6) недостаточно для достижения требуемой точности вычислений и необходимо удержать и исследовать дополнительные члены в правой части (6). Это и является целью настоящей работы. 


\section{2. УМЕРЕННО МАЛЫЕ ЭЙКОНАЛЬНЫЕ АМПЛИТУДЫ}

В нашем анализе мы ограничимся тремя членами ${ }^{2)}$ в разложении экспоненты $e^{i \chi}$ в уравнении (2):

$$
1-e^{i \chi}=-i\left(\chi-\frac{\chi^{3}}{6}\right)+\frac{\chi^{2}}{2}+\mathrm{O}\left(\chi^{4}\right) .
$$

При этом соответствующее приближение для амплитуды имеет вид

$$
A(s, t) \simeq\left[A_{1}(s, t)-A_{3}(s, t)\right]+i A_{2}(s, t),
$$

где

$$
\begin{aligned}
& A_{1}(s, t)=4 \pi s \int_{0}^{\infty} d b b J_{0}(b \sqrt{-t}) \chi(s, b), \\
& A_{2}(s, t)=2 \pi s \int_{0}^{\infty} d b b J_{0}(b \sqrt{-t}) \chi^{2}(s, b), \\
& A_{3}(s, t)=\frac{2}{3} \pi s \int_{0}^{\infty} d b b J_{0}(b \sqrt{-t}) \chi^{3}(s, b) .
\end{aligned}
$$

Опуская члены порядка $\mathrm{O}\left(\chi^{4}\right)$, находим, что

$$
\begin{aligned}
\frac{d \sigma(s, t)}{d t}= & \frac{1}{16 \pi s^{2}}\left\{\left|A_{1}(s, t)\right|^{2}-i\left[A_{1}(s, t) A_{2}^{*}(s, t)-A_{1}^{*}(s, t) A_{2}(s, t)\right]+\right. \\
& \left.+\left|A_{2}(s, t)\right|^{2}-\left[A_{1}(s, t) A_{3}^{*}(s, t)+A_{1}^{*}(s, t) A_{3}(s, t)\right]\right\} .
\end{aligned}
$$

В частности, если мнимая часть $A_{\mathrm{B}}(s, t)$ равна нулю, то дифференциальное сечение рассеяния в рассматриваемом приближении выглядит как

$$
\frac{d \sigma(s, t)}{d t}=\frac{1}{16 \pi s^{2}}\left\{A_{1}^{2}(s, t)+A_{2}^{2}(s, t)-2 A_{1}(s, t) A_{3}(s, t)\right\} .
$$

Для чисто мнимой борновской амплитуды величины $A_{1}$ и $A_{3}$ тоже являются чисто мнимыми, в то время как $A_{2}$ - вещественная величина, и мы видим, что

$$
\begin{aligned}
\frac{d \sigma(s, t)}{d t}= & \frac{1}{16 \pi s^{2}}\left\{A_{1}^{2}(s, t)-2 i A_{1}(s, t) A_{2}(s, t)+\right. \\
& \left.+A_{2}^{2}(s, t)+2 A_{1}(s, t) A_{3}(s, t)\right\} .
\end{aligned}
$$

Мы исследуем три части амплитуды (8)-(10) по отдельности. Учитывая формулы (А.1), (А.2), приведенные в приложении А, мы немедленно получаем из (8) выражение

$$
\begin{aligned}
A_{1}(s, t) & =\int_{0}^{\infty} d b b J_{0}(b \sqrt{-t}) \int_{0}^{\infty} d q_{\perp} q_{\perp} J_{0}\left(q_{\perp} b\right) A_{\mathrm{B}}\left(s, q_{\perp}\right)= \\
& =\int_{0}^{\infty} d q_{\perp} q_{\perp} A_{\mathrm{B}}\left(s, q_{\perp}\right) \int_{0}^{\infty} d b b J_{0}\left(q_{\perp} b\right) J_{0}(b \sqrt{-t})=A_{\mathrm{B}}(s, \sqrt{-t}) .
\end{aligned}
$$

2) По поводу удержания большего количества членов в правой части (7) см. замечания в конце этого раздела. 
Для изучения аналитических свойств амплитуды по переменной $t$ необходимо обобщить интеграл

$$
\int_{0}^{\infty} d x x J_{0}(a x) J_{0}(b x)
$$

на случай, когда $a, b$ являются комплексными числами ${ }^{3)}$. Это сделано в приложении А (см. формулы (А.3), (А.4)).

Вторую часть амплитуды (9) можно исследовать, используя уравнения (А.5), (А.6) из приложения А. В результате мы получаем, что

$$
\begin{aligned}
A_{2}(s, t)= & \frac{1}{8 \pi s} \int_{0}^{\infty} d b b J_{0}(b \sqrt{-t}) \int_{0}^{\infty} d q_{\perp} q_{\perp} J_{0}\left(q_{\perp} b\right) A_{\mathrm{B}}\left(s, q_{\perp}\right) \times \\
& \times \int_{0}^{\infty} d q_{\perp}^{\prime} q_{\perp}^{\prime} J_{0}\left(q_{\perp}^{\prime} b\right) A_{\mathrm{B}}\left(s, q_{\perp}^{\prime}\right)=\frac{1}{8 \pi s} \int_{0}^{\infty} d q_{\perp} q_{\perp} A_{\mathrm{B}}\left(s, q_{\perp}\right) \times \\
& \times \int_{0}^{\infty} d q_{\perp}^{\prime} q_{\perp}^{\prime} A_{\mathrm{B}}\left(s, q_{\perp}^{\prime}\right) \int_{0}^{\infty} d b b J_{0}\left(q_{\perp} b\right) J_{0}\left(q_{\perp}^{\prime} b\right) J_{0}(b \sqrt{-t})= \\
= & \frac{1}{4 \pi^{2} s} \int_{0}^{\infty} d q_{\perp} q_{\perp} A_{\mathrm{B}}\left(s, q_{\perp}\right) \int_{0}^{\infty} d q_{\perp}^{\prime} q_{\perp}^{\prime} A_{\mathrm{B}}\left(s, q_{\perp}^{\prime}\right) \theta\left(\sqrt{-t}-\left|q_{\perp}-q_{\perp}^{\prime}\right|\right) \times \\
& \times \theta\left(q_{\perp}+q_{\perp}^{\prime}-\sqrt{-t}\right) \frac{1}{\sqrt{\left[-t-\left(q_{\perp}-q_{\perp}^{\prime}\right)^{2}\right]\left[\left(q_{\perp}+q_{\perp}^{\prime}\right)^{2}+t\right]}} .
\end{aligned}
$$

Определим новые переменные

$$
q_{1}=q_{\perp}+q_{\perp}^{\prime}, \quad q_{2}=q_{\perp}-q_{\perp}^{\prime}
$$

В этих переменных формулу для $A_{2}(s, t)$ можно переписать следующим образом:

$$
\begin{aligned}
A_{2}(s, t)= & \frac{1}{32 \pi^{2} s} \int_{0}^{\infty} d q_{1} \int_{-q_{1}}^{q_{1}} d q_{2} A_{\mathrm{B}}\left(s, \frac{q_{1}+q_{2}}{2}\right) A_{\mathrm{B}}\left(s, \frac{q_{1}-q_{2}}{2}\right) \times \\
& \times \theta\left(q_{1}-\sqrt{-t}\right) \theta\left(\sqrt{-t}-\left|q_{2}\right|\right) \frac{\left(q_{1}^{2}-q_{2}^{2}\right)}{\sqrt{\left(-t-q_{2}^{2}\right)\left(q_{1}^{2}+t\right)}}= \\
= & \frac{1}{16 \pi^{2} s} \int_{\sqrt{-t}}^{\infty} d q_{1} \int_{0}^{\sqrt{-t}} d q_{2} A_{\mathrm{B}}\left(s, \frac{q_{1}+q_{2}}{2}\right) A_{\mathrm{B}}\left(s, \frac{q_{1}-q_{2}}{2}\right) \times \\
& \times \frac{\left(q_{1}^{2}-q_{2}^{2}\right)}{\sqrt{\left(-t-q_{2}^{2}\right)\left(q_{1}^{2}+t\right)}} .
\end{aligned}
$$

После еще одной замены переменных $q_{i}=x_{i} \sqrt{-t}, i=1,2$, мы приходим к окончательному выражению для $A_{2}(s, t)$ :

$$
\begin{aligned}
A_{2}(s, t)= & \frac{1}{16 \pi^{2}} \frac{-t}{s} \int_{1}^{\infty} d x_{1} \int_{0}^{1} d x_{2} \frac{x_{1}^{2}-x_{2}^{2}}{\sqrt{\left(x_{1}^{2}-1\right)\left(1-x_{2}^{2}\right)}} \times \\
& \times A_{\mathrm{B}}\left(s, \frac{\sqrt{-t}\left(x_{1}+x_{2}\right)}{2}\right) A_{\mathrm{B}}\left(s, \frac{\sqrt{-t}\left(x_{1}-x_{2}\right)}{2}\right) .
\end{aligned}
$$

\footnotetext{
3) Строго говоря, достаточно рассмотреть положительные $a$ и комплексные $b$.
} 
Для третьей части амплитуды (10) мы получаем формулу

$$
\begin{aligned}
A_{3}(s, t)= & \frac{1}{96 \pi^{2} s^{2}} \int_{0}^{\infty} d b b J_{0}(b \sqrt{-t}) \int_{0}^{\infty} d q_{\perp} q_{\perp} J_{0}\left(q_{\perp} b\right) A_{\mathrm{B}}\left(s, q_{\perp}\right) \times \\
& \times \int_{0}^{\infty} d q_{\perp}^{\prime} q_{\perp}^{\prime} J_{0}\left(q_{\perp}^{\prime} b\right) A_{\mathrm{B}}\left(s, q_{\perp}^{\prime}\right) \int_{0}^{\infty} d q_{\perp}^{\prime \prime} q_{\perp}^{\prime \prime} J_{0}\left(q_{\perp}^{\prime \prime} b\right) A_{\mathrm{B}}\left(s, q_{\perp}^{\prime \prime}\right)= \\
= & \frac{1}{96 \pi^{2} s^{2}} \int_{0}^{\infty} d q_{\perp} q_{\perp} A_{\mathrm{B}}\left(s, q_{\perp}\right) \int_{0}^{\infty} d q_{\perp}^{\prime} q_{\perp}^{\prime} A_{\mathrm{B}}\left(s, q_{\perp}^{\prime}\right) \int_{0}^{\infty} d q_{\perp}^{\prime \prime} q_{\perp}^{\prime \prime} A_{\mathrm{B}}\left(s, q_{\perp}^{\prime \prime}\right) \times \\
& \times \int_{0}^{\infty} d b b J_{0}\left(q_{\perp} b\right) J_{0}\left(q_{\perp}^{\prime} b\right) J_{0}\left(q_{\perp}^{\prime \prime} b\right) J_{0}(b \sqrt{-t}) .
\end{aligned}
$$

Используя уравнения (А.13), (А.20) из приложения А, представим выражение (14) для $A_{3}(s, t)$ в виде:

$$
\begin{aligned}
A_{3}(s, t)= & \frac{1}{96 \pi^{2}}\left(\frac{-t}{s}\right)^{2} \int_{0}^{\infty} d x A_{\mathrm{B}}(s, \sqrt{-t} x) \int_{0}^{\infty} d x^{\prime} A_{\mathrm{B}}\left(s, \sqrt{-t} x^{\prime}\right) \times \\
& \times \int_{0}^{\infty} d x^{\prime \prime} A_{\mathrm{B}}\left(s, \sqrt{-t} x^{\prime \prime}\right) G\left(x, x^{\prime}, x^{\prime \prime}\right) .
\end{aligned}
$$

Здесь введена функция

$$
G\left(x, x^{\prime}, x^{\prime \prime}\right)= \begin{cases}\frac{1}{\pi^{2} A} K\left(\frac{\sqrt{B}}{A}\right), & A^{2}>B, \\ \frac{1}{\pi^{2} \sqrt{B}} K\left(\frac{A}{\sqrt{B}}\right), & 0 \leqslant A^{2}<B, \\ 0, & A^{2}<0,\end{cases}
$$

где $K(k)$ - полный эллиптический интеграл первого рода (см. формулу (А.16)) и использованы обозначения

$$
16 A^{2}=\left[\left(x^{\prime \prime}+1\right)^{2}-\left(x-x^{\prime}\right)^{2}\right]\left[\left(x+x^{\prime}\right)^{2}-\left(x^{\prime \prime}-1\right)^{2}\right], \quad B=x x^{\prime} x^{\prime \prime} .
$$

Определим новые переменные

$$
x_{1}=x+x^{\prime}, \quad x_{2}=x-x^{\prime}, \quad x_{3}=x^{\prime \prime},
$$

которые пробегают область, определенную следующими неравенствами:

$$
\begin{gathered}
0 \leqslant x_{1} \leqslant \infty, \quad-x_{1} \leqslant x_{2} \leqslant x_{1}, \quad 0 \leqslant x_{3} \leqslant \infty, \\
{\left[\left(x_{3}+1\right)^{2}-x_{2}^{2}\right]\left[x_{1}^{2}-\left(x_{3}-1\right)^{2}\right]>0 .}
\end{gathered}
$$

Тогда из уравнений (15)-(20) находим, что

$$
\begin{aligned}
A_{3}(s, t)= & \frac{1}{96 \pi^{2}}\left(\frac{-t}{s}\right)^{2} \int_{0}^{\infty} d x_{1} \int_{-x_{1}}^{x_{1}} d x_{2} \int_{0}^{\infty} d x_{3} \theta\left(\left[\left(x_{3}+1\right)^{2}-x_{2}^{2}\right]\left[x_{1}^{2}-\left(x_{3}-1\right)^{2}\right]\right) \times \\
& \times H\left(s, t ; x_{1}, x_{2}, x_{3}\right),
\end{aligned}
$$


где

$$
\begin{aligned}
H\left(s, t ; x_{1}, x_{2}, x_{3}\right)= & A_{\mathrm{B}}\left(s, \frac{\sqrt{-t}\left(x_{1}+x_{2}\right)}{2}\right) A_{\mathrm{B}}\left(s, \frac{\sqrt{-t}\left(x_{1}-x_{2}\right)}{2}\right) \times \\
& \times A_{\mathrm{B}}\left(s, \sqrt{-t} x_{3}\right) G\left(\frac{x_{1}+x_{2}}{2}, \frac{x_{1}-x_{2}}{2}, x_{3}\right) .
\end{aligned}
$$

Используя формулу (Б.2) из приложения Б, получаем выражение

$$
\begin{aligned}
A_{3}(s, t)= & \frac{1}{96 \pi^{2}}\left(\frac{-t}{s}\right)^{2}\left[\int_{0}^{1} d x_{1} \int_{0}^{x_{1}} d x_{2} \int_{1-x_{1}}^{x_{1}+1} d x_{3}+\int_{1}^{2} d x_{1} \int_{0}^{1} d x_{2} \int_{0}^{x_{1}+1} d x_{3}+\right. \\
& +\int_{1}^{2} d x_{1} \int_{1}^{x_{1}} d x_{2} \int_{\left|x_{2}\right|-1}^{x_{1}+1} d x_{3}+\int_{2}^{\infty} d x_{1} \int_{0}^{x_{1}+1} d x_{2} \int_{0}^{x_{1}+1} d x_{3}+ \\
& +\int_{2}^{\infty} d x_{1} \int_{1}^{x_{1}} d x_{2} \int_{\left|x_{2}\right|-1}^{\left.x_{3}\right]}\left[H\left(s, t ; x_{1}, x_{2}, x_{3}\right)+H\left(s, t ; x_{1},-x_{2}, x_{3}\right)\right] .
\end{aligned}
$$

Формулы (11)-(13) и (21) можно применить для вычисления дифференциальных сечений при фиксированных $s$ и $t$ при условии, что борновская амплитуда $A_{\mathrm{B}}(s, t)$ известна и аппроксимация (7) оправданна. Преимущество полученных интегралов заключается в том, что они не содержат быстро осциллирующих подынтегральных выражений $^{4)}$. Конечно, при этом предполагается, что борновская амплитуда не содержит таких осциллирующих выражений по переменной $t$.

Если требуется удержать больше слагаемых в разложении (7), необходимо использовать интегралы с более чем четырьмя функциями Бесселя. Соответствующие формулы представлены в приложении А (см. формулы (А.22), (А.23)).

\section{ПРИЛОЖЕНИЕ А}

В данном приложении рассмотрены несобственные интегралы первого рода, которые содержат произведения функций Бесселя. Хорошо известная формула с двумя функциями $J_{\nu}(z)$ обобщена на нецелые значения $\nu$. Уточнено аналитическое выражение для интеграла с четырьмя функциями $J_{0}(z)$. Наконец, получены выражения для интегралов, содержащих произведения пяти и шести функций $J_{0}(z)$.

Рассмотрим сначала интеграл с двумя функциями Бесселя $J_{n}(z)$ :

$$
F_{2}^{(n)}(a, b)=\int_{0}^{\infty} d x x J_{n}(a x) J_{n}(b x), \quad n=0,1, \ldots
$$

Как известно, при $a, b>0$ он равен (см. книги [14], [15])

$$
F_{2}^{(n)}(a, b)=\frac{1}{a} \delta(a-b) .
$$

Вычислим более общий интеграл, содержащий функции Бесселя с нецелыми индексами $\nu$ (см. задачу 3.16(а) в книге [14]):

$$
F_{2}^{(\nu)}(a, b)=\int_{0}^{\infty} d x x J_{\nu}(a x) J_{\nu}(b x) .
$$

\footnotetext{
4) Полный эллиптический интеграл первого рода $K(k)$ в выражении (16) есть монотонно возрастающая функция модуля $k(0 \leqslant k<1)$.
} 
В отличие от уравнения (А.1) мы не будем предполагать, что $a$ и $b$ - положительные числа. Начнем интегрирование с вычисления второго экспоненциального интеграла Вебера (см., например, книгу [16]):

$$
I_{\mathrm{W}}(a, b ; p)=\int_{0}^{\infty} d x x e^{-p^{2} x^{2}} J_{\nu}(a x) J_{\nu}(b x)=\frac{1}{2 p^{2}} e^{-\left(a^{2}+b^{2}\right) / 4 p^{2}} I_{0}\left(\frac{a b}{2 p^{2}}\right),
$$

где $I_{\nu}(z)$ - модифицированная функция Бесселя первого рода. Данная формула справедлива при $|\arg p|<\pi / 4, \operatorname{Re} \nu>-1$. Параметры $a$ и $b$ могут быть произвольными комплексными числами.

Пусть в дальнейшем $p>0$. При условии $|\arg a b|<\pi / 2$ можно использовать асимптотическую формулу из книги [16]

$$
\left.I_{\nu}\left(\frac{a b}{2 p^{2}}\right)\right|_{p \rightarrow 0}=\frac{1}{\sqrt{\pi a b / p^{2}}} e^{a b / 2 p^{2}}\left[1+\mathrm{O}\left(p^{2}\right)\right]
$$

и получить выражение

$$
\left.I_{\mathrm{W}}(a, b ; p)\right|_{p \rightarrow 0}=\frac{1}{\sqrt{a b}} \frac{1}{2 \sqrt{\pi p^{2}}} e^{-(a-b)^{2} / 4 p^{2}}\left[1+\mathrm{O}\left(p^{2}\right)\right] .
$$

Известно [17], что при $t>0$

$$
\left.\frac{1}{2 \sqrt{\pi t}} e^{-x^{2} / 4 t}\right|_{t \rightarrow 0} \rightarrow \delta(x)
$$

В результате мы приходим к обобщению формулы (А.2) на случай $\operatorname{Re} \nu>-1$, $|\arg a b|<\pi / 2$ :

$$
F_{2}^{(\nu)}(a, b)=\left.I_{\mathrm{W}}(a, b ; p)\right|_{p \rightarrow 0}=\frac{1}{\sqrt{a b}} \delta(a-b) .
$$

Интеграл с тремя функциями Бесселя $J_{0}(z)$

$$
F_{3}(a, b, c)=\int_{0}^{\infty} d x x J_{0}(a x) J_{0}(b x) J_{0}(c x),
$$

где $a, b, c>0$, дается формулой (см., например, книги [16], [18])

$$
F_{3}(a, b, c)= \begin{cases}\frac{1}{2 \pi \Delta_{3}}, & \Delta_{3}^{2}>0 \\ 0, & \Delta_{3}^{2}<0\end{cases}
$$

в которой

$$
16 \Delta_{3}^{2}=\left[c^{2}-(a-b)^{2}\right]\left[(a+b)^{2}-c^{2}\right] .
$$

Заметим, что выражение (А.6) не определено, если $\Delta_{3}^{2}=0$ (последнее обстоятельство имеет место, если один из параметров равен сумме остальных, например $c=a+b>0)$.

Согласуется ли формула (А.6) с (А.2)? Для того чтобы оценить выражение (А.6) в пределе $c=0$, положим $c=\varepsilon \ll 1$ (считая $a, b>0$ ). Тогда получаем, что

$$
\left.\frac{1}{2 \pi \Delta_{3}}\right|_{c=\varepsilon \ll 1} \simeq \frac{2}{(a+b)} \frac{1}{\pi \sqrt{\varepsilon^{2}-(a-b)^{2}}} .
$$


Рассмотрим функцию

$$
f_{\varepsilon}(x)=\theta(\varepsilon+x) \theta(\varepsilon-x) \frac{1}{\pi \sqrt{\varepsilon^{2}-x^{2}}} .
$$

Из (А.8) находим, что

$$
\lim _{\varepsilon \rightarrow 0} \int_{\alpha}^{\beta} d x f_{\varepsilon}(x)=\left\{\begin{array}{ll}
0, & \alpha<\beta<0 \\
1, & \alpha<0<\beta .
\end{array} \text { или } 0<\alpha<\beta,\right.
$$

Покажем, что функция $f_{\varepsilon}(x)$ является дельтаобразной последовательностью. Для этого рассмотрим последовательность первообразных функций

$$
F_{\varepsilon}(x)=\int_{-1}^{x} f_{\varepsilon}(z) d z .
$$

В пределе $\varepsilon \rightarrow 0$ функция $F_{\varepsilon}(x)$ стремится к постоянной, равной нулю при $x<0$ и единице при $x>0$. В то же самое время она равномерно по $\varepsilon$ ограничена в каждом промежутке. Следовательно, справедливы пределы

$$
\left.F_{\varepsilon}(x)\right|_{\varepsilon \rightarrow 0}=\theta(x)
$$

и соответственно

$$
\left.f_{\varepsilon}(x)\right|_{\varepsilon \rightarrow 0}=\theta^{\prime}(x)=\delta(x) .
$$

В результате в пределе $c=0$ мы получаем формулу (А.2) (при $n=0$ ) из формулы (А.6).

Рассмотрим теперь интеграл с четьръмя функциями Бесселя $J_{0}(z)$ :

$$
F_{4}(a, b, c, d)=\int_{0}^{\infty} d x x J_{0}(a x) J_{0}(b x) J_{0}(c x) J_{0}(d x),
$$

где $a, b, c, d>0$. В книге [18] приведена следующая формула:

$$
F_{4}(a, b, c, d)= \begin{cases}\frac{1}{\pi^{2} \Delta_{4}} K\left(\frac{\sqrt{a b c d}}{\Delta_{4}}\right), & \Delta_{4}^{2}>a b c d, \\ \frac{1}{\pi^{2} \sqrt{a b c d}} K\left(\frac{\Delta_{4}}{\sqrt{a b c d}}\right), & \Delta_{4}^{2}<a b c d,\end{cases}
$$

где введено обозначение

$$
16 \Delta_{4}^{2}=(a+b+c-d)(a+b+d-c)(a+c+d-b)(b+c+d-a),
$$

a

$$
K(k)=\int_{0}^{\pi / 2} \frac{d \theta}{\sqrt{1-k^{2} \sin ^{2} \theta}}=\int_{0}^{1} \frac{d t}{\sqrt{\left(1-t^{2}\right)\left(1-k^{2} t^{2}\right)}}
$$

есть полный эллиптический интеграл первого рода $(0 \leqslant k<1)$ [19]. Для $\Delta_{4}^{2}=a b c d$ интеграл (А.13) не определен. Действительно, функция $K(k)$ имеет точку ветвления $k=1$ и расходится [19] при $k \rightarrow 1$ как

$$
\left.K(k)\right|_{k \rightarrow 1} \simeq \ln \left(\frac{4}{\sqrt{1-k^{2}}}\right) .
$$


K сожалению, формула (А.14) не включает в себя один важный ${ }^{5)}$ случай: $\Delta_{4}^{2}<0$. Проанализируем его детально и покажем, что $F_{4}(a, b, c, d)=0$ при $\Delta_{4}^{2}<0$. Для этого используем интегральное представление функций Бесселя ${ }^{6)}$ [20]:

$$
J_{n}(z)=\frac{1}{2 \pi} \int_{0}^{2 \pi} d \varphi e^{i(z \sin \varphi-n \varphi)}, \quad n=0,1,2 \ldots
$$

Тогда получим, что

$$
\begin{aligned}
F_{4}(a, b, c, d)= & \frac{1}{(2 \pi)^{4}} \int_{0}^{\infty} d B B \int_{0}^{2 \pi} d \varphi_{1} \int_{0}^{2 \pi} d \varphi_{2} \int_{0}^{2 \pi} d \varphi_{3} \int_{0}^{2 \pi} d \varphi_{4} \times \\
& \times e^{i B\left(a \sin \varphi_{1}+b \sin \varphi_{2}+c \sin \varphi_{3}+d \sin \varphi_{4}\right.}= \\
= & \frac{1}{(2 \pi)^{5}} \int_{0}^{2 \pi} d \varphi_{1} \int_{0}^{2 \pi} d \varphi_{2} \int_{0}^{2 \pi} d \varphi_{3} \int_{0}^{2 \pi} d \varphi_{4} \int_{0}^{\infty} d \vec{B} \times \\
& \times e^{i \vec{B}(\vec{a}+\vec{b}+\vec{c}+\vec{d})}
\end{aligned}
$$

где $\vec{B}=(0, B)$ и введены двумерные векторы

$$
\begin{aligned}
\vec{a} & =\left(a \cos \varphi_{1}, a \sin \varphi_{1}\right), & \vec{b} & =\left(b \cos \varphi_{2}, b \sin \varphi_{2}\right), \\
\vec{c} & =\left(c \cos \varphi_{3}, c \sin \varphi_{3}\right), & \vec{d} & =\left(d \cos \varphi_{4}, d \sin \varphi_{4}\right) .
\end{aligned}
$$

Taк как

$$
\begin{aligned}
\frac{1}{(2 \pi)^{2}} \int_{0}^{\infty} d \vec{B} e^{i \vec{B}(\vec{a}+\vec{b}+\vec{c}+\vec{d})}= & \delta^{(2)}(\vec{a}+\vec{b}+\vec{c}+\vec{d})= \\
= & \delta\left(a \sin \varphi_{1}+b \sin \varphi_{2}+c \sin \varphi_{3}+d \sin \varphi_{4}\right) \times \\
& \times \delta\left(a \cos \varphi_{1}+b \cos \varphi_{2}+c \cos \varphi_{3}+d \cos \varphi_{4}\right)
\end{aligned}
$$

мы находим, что

$$
\begin{aligned}
F_{4}(a, b, c, d)= & \frac{1}{(2 \pi)^{3}} \int_{0}^{2 \pi} d \varphi_{1} \int_{0}^{2 \pi} d \varphi_{2} \int_{0}^{2 \pi} d \varphi_{3} \int_{0}^{2 \pi} d \varphi_{4} \times \\
& \times \delta\left(a \sin \varphi_{1}+b \sin \varphi_{2}+c \sin \varphi_{3}+d \sin \varphi_{4}\right) \times \\
& \times \delta\left(a \cos \varphi_{1}+b \cos \varphi_{2}+c \cos \varphi_{3}+d \cos \varphi_{4}\right) .
\end{aligned}
$$

Из определения (А.15) нетрудно видеть, что условие $\Delta_{4}^{2}<0$ реализуется только тогда, когда один из параметров $a, b, c, d$ больше суммы остальных. Без потери общности можно положить $d>a+b+c$. Интеграл (А.17) отличен от нуля, только если

$$
\begin{aligned}
a \sin \varphi_{1}+b \sin \varphi_{2}+c \sin \varphi_{3}+d \sin \varphi_{4} & =0, \\
a \cos \varphi_{1}+b \cos \varphi_{2}+c \cos \varphi_{3}+d \cos \varphi_{4} & =0 .
\end{aligned}
$$

В частности, это означает выполнение равенств

$$
\begin{aligned}
1= & \sin ^{2} \varphi_{4}+\cos ^{2} \varphi_{4}=\frac{1}{d^{2}}\left[a^{2}+b^{2}+c^{2}+2 a b \cos \left(\varphi_{1}-\varphi_{2}\right)+\right. \\
& \left.+2 b c \cos \left(\varphi_{2}-\varphi_{3}\right)+2 c a \cos \left(\varphi_{3}-\varphi_{1}\right)\right]=A(a, b, c) .
\end{aligned}
$$

5) Если формально использовать вторую строку в правой части уравнения (А.14) для отрицательных значений $\Delta_{4}^{2}$, получится, как мы увидим, неверный результат.

6) Это представление было получено Ф. В. Бесселем. 
Но из очевидного неравенства

$$
A(a, b, c) \leqslant \frac{(a+b+c)^{2}}{d^{2}}<1
$$

следует, что оба уравнения (А.18) не могут быть справедливы одновременно, и мы приходим к соотношению ${ }^{7}$

$$
F_{4}(a, b, c, d)=0, \quad \Delta_{4}^{2}<0
$$

что и требовалось доказать.

Осталось показать, что интеграл $F_{4}(a, b, c, d)\left(\right.$ А.13) определен при $\Delta_{4}^{2}=0$ (подразумевается, что $a, b, c, d>0)$. Заметим, что условие $\Delta_{4}^{2}=0$ выполняется, если только один из параметров $a, b, c, d$ равен сумме остальных трех. Пусть $d=a+b+c$. Асимптотическая формула для функции Бесселя [20]

$$
\left.J_{0}(z)\right|_{|z| \gg 1}=\sqrt{\frac{2}{\pi z}} \cos \left(z-\frac{\pi}{4}\right)\left[1+\mathrm{O}\left(|z|^{-1}\right)\right], \quad|\arg z|<\pi,
$$

позволяет получить при $x>0$ разложение

$$
\begin{aligned}
\left.J_{0}(a x) J_{0}(b x) J_{0}(c x) J_{0}[(a+b+c) x]\right|_{x \gg 1}=\frac{1}{2 \pi^{2} x^{2} \sqrt{a b c(a+b+c)}} \times \\
\quad \times\{\cos (2 a x)+\cos (2 b x)+\cos (2 c x)+\sin [2(a+b) x]+\sin [2(b+c) x]+ \\
+\sin [2(a+c) x]\}\left[1+\mathrm{O}\left(x^{-1}\right)\right]=J_{\text {asym }}(x)+\mathrm{O}\left(x^{-3}\right) .
\end{aligned}
$$

Тогда мы можем записать для некоторого фиксированного $x_{0}>0$, что

$$
\begin{aligned}
\left.F_{4}(a, b, c, d)\right|_{d=a+b+c}= & \int_{0}^{x_{0}} d x x J_{0}(a x) J_{0}(b x) J_{0}(c x) J_{0}[(a+b+c) x]+ \\
& +\int_{x_{0}}^{\infty} d x x\left\{J_{0}(a x) J_{0}(b x) J_{0}(c x) J_{0}[(a+b+c) x]-J_{\text {asym }}(x)\right\}+ \\
& +\int_{x_{0}}^{\infty} d x x J_{\text {asym }}(x) .
\end{aligned}
$$

Первый интеграл в (А.19), очевидно, хорошо определен. Во втором интеграле подынтегральное выражение быстро убывает как $\mathrm{O}\left(x^{-2}\right)$ при $x \rightarrow \infty$. Наконец, третий интеграл в (А.19) есть линейная комбинация интегральных синусов

$$
\operatorname{Si}(z)=-\int_{z}^{\infty} \frac{\sin t}{t} d t
$$

в которых $z=2(a+b) x_{0}, 2(b+c) x_{0}$ или $2(a+c) x_{0}$, и интегральных косинусов

$$
\operatorname{Ci}(z)=-\int_{z}^{\infty} \frac{\cos t}{t} d t
$$

в которых $z=2 a x_{0}, 2 b x_{0}$, или $2 c x_{0}$. Так как $\operatorname{Si}(z)$ и $\operatorname{Ci}(z)$ являются хорошо определенными функциями при $z>0$ [20], заключаем, что интеграл $F_{4}(a, b, c, d)(\mathrm{A} .13)$ сходится при $\Delta_{4}^{2}=0$.

\footnotetext{
7) Таким же образом можно показать, что $F_{3}(a, b, c)=0$ для $\Delta_{3}^{2}<0$.
} 
Итак, мы показали, что формулу (А.14) можно обобщить следующим образом при $a, b, c, d>0$ :

$$
F_{4}(a, b, c, d)= \begin{cases}\frac{1}{\pi^{2} \Delta_{4}} K\left(\frac{\sqrt{a b c d}}{\Delta_{4}}\right), & \Delta_{4}^{2}>a b c d \\ \frac{1}{\pi^{2} \sqrt{a b c d}} K\left(\frac{\Delta_{4}}{\sqrt{a b c d}}\right), & 0<\Delta_{4}^{2}<a b c d, \\ \frac{1}{2 \pi \sqrt{a b c d}}, & \Delta_{4}^{2}=0 \\ 0, & \Delta_{4}^{2}<0 .\end{cases}
$$

Здесь учтено, что $K(0)=\pi / 2$. Заметим, что

$$
16 \Delta_{4}^{2}=\left[(c+d)^{2}-(a-b)^{2}\right]\left[(a+b)^{2}-(c-d)^{2}\right] .
$$

Следовательно, $\Delta_{4}^{2} \rightarrow \Delta_{3}^{2}$ в пределе $d=0$ и формула (А.20) (см. первую строку в ее правой части) переходит в формулу (А.6). Соответственно в пределе $c=d=0$ выражение (А.20) трансформируется в выражение (А.2). Чтобы продемонстрировать это, достаточно положить $c+d=\varepsilon \ll 1$ и затем применить аргументы, использованные при выводе формул (А.7)-(А.12).

Таким образом, мы показали, что формулы (А.2), (А.6) и (А.20) образуют непротиворечивый набор уравнений в том смысле, что

$\left.F_{4}(a, b, c, d)\right|_{d=0}=F_{3}(a, b, c),\left.\quad F_{4}(a, b, c, d)\right|_{c=d=0}=F_{2}(a, b),\left.\quad F_{3}(a, b, c)\right|_{c=0}=F_{2}(a, b)$.

Подчеркнем, что это утверждение справедливо для соотношений между соответствующими аналитическими выражениями, полученными для ${ }^{8)} F_{4}(a, b, c, d)(\mathrm{A} .20)$, $F_{3}(a, b, c)(\mathrm{A} .6)$ и $F_{2}(a, b)(\mathrm{A} .2)$.

Расмотрим теперь интеграл с пятью функциями Бесселя $J_{0}(z)$ (как и ранее, $a, b, c, d, e>0)$ :

$$
F_{5}(a, b, c, d, e)=\int_{0}^{\infty} d x x J_{0}(a x) J_{0}(b x) J_{0}(c x) J_{0}(d x) J_{0}(e x) .
$$

Запишем тождество

$$
J_{0}(a x) J_{0}(b x)=\int_{0}^{\infty} d y \delta(x-y) J_{0}(a y) J_{0}(b y) .
$$

Используя формулу (А.2) и равенство

$$
\delta(x-y)=y \int_{0}^{\infty} d t t J_{0}(t x) J_{0}(t y)
$$

получим из (А.21), что

$$
J_{0}(a x) J_{0}(b x)=\int_{0}^{\infty} d t t J_{0}(t x) \int_{0}^{\infty} d y y J_{0}(a y) J_{0}(b y) J_{0}(t y) .
$$

8)Что касается самих интегралов (А.1), (А.5) и (А.13), подобные соотношения между ними являются очевидными, если учесть, что $J_{0}(0)=1$. 
Таким образом, справедливо соотношение

$$
\begin{aligned}
F_{5}(a, b, c, d, e)= & \int_{0}^{\infty} d t t \int_{0}^{\infty} d x x J_{0}(c x) J_{0}(d x) J_{0}(e x) J_{0}(t x) \times \\
& \times \int_{0}^{\infty} d y y J_{0}(a y) J_{0}(b y) J_{0}(t y) .
\end{aligned}
$$

В результате мы приходим к следующему выражению:

$$
F_{5}(a, b, c, d, e)=\int_{0}^{\infty} d t t F_{3}(a, b, t) F_{4}(c, d, e, t),
$$

где функции $F_{3}(a, b, t)$ и $F_{4}(c, d, e, t)$ определены соответственно формулами (А.6) и (А.20).

Аналогично получаем, что при $a, b, c, d, e, f>0$

$$
\begin{aligned}
F_{6}(a, b, c, d, e, f) & =\int_{0}^{\infty} d x x J_{0}(a x) J_{0}(b x) J_{0}(c x) J_{0}(d x) J_{0}(e x) J_{0}(f x)= \\
& =\int_{0}^{\infty} d t t F_{3}(a, b, t) \int_{0}^{\infty} d q q F_{3}(c, d, q) \int_{0}^{\infty} d p p F_{3}(e, f, p) F_{3}(t, q, p)= \\
& =\int_{0}^{\infty} d t t F_{4}(a, b, c, t) F_{4}(d, e, f, t) .
\end{aligned}
$$

Аналитическое выражение для $F_{4}\left(a_{1}, a_{2}, a_{3}, a_{4}\right)$ следует взять из (А.20).

Действуя таким же образом, можно выразить интеграл общего вида

$$
F_{n}\left(a_{1}, \ldots, a_{n}\right)=\int_{0}^{\infty} d x x \prod_{k=1}^{n} J_{0}\left(a_{k} x\right)
$$

где $n>6$ и $a_{k}>0, k=1, \ldots, n$, через $(n-3)$-мерный интеграл от алгебраических функций. Заметим при этом, что $F_{n}\left(a_{1}, \ldots, a_{n}\right)=0$, если $a_{n}>a_{1}+a_{2}+\cdots+a_{n-1}$.

\section{ПРИЛОЖЕНИЕ Б}

В данном приложении мы исследуем возможные ограничения на переменные $x_{1}$, $x_{2}, x_{3}$ в интеграле $(21)$, которые вытекают из неравенства (20)

$$
\left[\left(x_{3}+1\right)^{2}-x_{2}^{2}\right]\left[x_{1}^{2}-\left(x_{3}-1\right)^{2}\right]>0 .
$$

Напомним также, что (см. уравнение (19))

$$
0 \leqslant x_{1} \leqslant \infty, \quad-x_{1} \leqslant x_{2} \leqslant x_{1}, \quad 0 \leqslant x_{3} \leqslant \infty .
$$

Если $0 \leqslant x_{3}<1$, из неравенства $(20)$ следуют неравенства

$$
\max \left(\left|x_{2}\right|-1,1-x_{1}, 0\right) \leqslant x_{3}<1 \quad \text { или } \quad 0 \leqslant x_{3} \leqslant \min \left(\left|x_{2}\right|-1,1-x_{1}\right) \text {. }
$$


Аналогично, при $x_{3} \geqslant 1$ из формулы (20) вытекает, что

$$
\max \left(\left|x_{2}\right|-1,1\right) \leqslant x_{3} \leqslant 1+x_{1} \quad \text { или } \quad 1+x_{1} \leqslant x_{3} \leqslant\left|x_{2}\right|-1 .
$$

Но так как $1+x_{1}>1-\left|x_{2}\right|$, в (Б.1) остается лишь первое неравенство

$$
\max \left(\left|x_{2}\right|-1,1\right) \leqslant x_{3} \leqslant 1+x_{1} .
$$

Пусть $H\left(x_{1}, x_{2}, x_{3}\right)$ - интегрируемая функция, тогда справедливо следующее выражение:

$$
\begin{aligned}
\int_{0}^{\infty} d x_{1} \int_{-x_{1}}^{x_{1}} d x_{2} \int_{0}^{\infty} d x_{3} \theta\left(\left[\left(x_{3}+1\right)^{2}-x_{2}^{2}\right]\left[x_{1}^{2}-\left(x_{3}-1\right)^{2}\right]\right) H\left(x_{1}, x_{2}, x_{3}\right)= \\
=\left[\int_{0}^{1} d x_{1} \int_{0}^{x_{1}} d x_{2} \int_{1-x_{1}}^{x_{1}+1} d x_{3}+\int_{1}^{2} d x_{1} \int_{0}^{1} d x_{2} \int_{0}^{x_{1}+1} d x_{3}+\right. \\
+\int_{1}^{2} d x_{1} \int_{1}^{x_{1}} d x_{2} \int_{\left|x_{2}\right|-1}^{x_{1}+1} d x_{3}+\int_{2}^{\infty} d x_{1} \int_{0}^{1} d x_{2} \int_{0}^{x_{1}+1} d x_{3}+ \\
\left.+\int_{2}^{\infty} d x_{1} \int_{1}^{x_{1}} d x_{2} \int_{\left|x_{2}\right|-1}^{x_{1}+1} d x_{3}\right]\left[H\left(x_{1}, x_{2}, x_{3}\right)+H\left(x_{1},-x_{2}, x_{3}\right)\right] .
\end{aligned}
$$

Благодарности. Автор признателен В.А. Петрову и В.Е. Рочеву за ценные замечания.

\section{Список литературы}

[1] G. Molière, Z. Naturforsch. A, 2:3 (1947), 133-145; Z. Naturforsch. A, 3:2 (1948), 78-97.

[2] R. J. Glauber, "High-energy collision theory", Lectures in Theoretical Physics, v. 1 (University of Colorado, Boulder, 1958), eds. W. E. Brittin, L. G. Dunham, Interscience, New York, 1959, 315-414.

[3] R. G. Newton, Scattering Theory of Waves and Particles, McGraw-Hill, New York, 1966.

[4] H. Cheng, T. T. Wu, Phys. Rev. Lett., 22:13 (1969), 666-669; Phys. Rev., 186:5 (1969), 1611-1618.

[5] A. A. Logunov, A. N. Tavkhelidze, Nuovo Cimento, 29:2 (1963), 380-389.

[6] V.R. Garsevanishvili, V.A. Matveev, L. A. Slepchenko, A. N. Tavkhelidze, Phys. Lett. B, 29:3 (1969), 191-192.

[7] V.A. Petrov, "High-energy implications of extended unitarity", Proceedings of 7th Conference (Blois Workshop) on Elastic and Diffractive Scattering (Blois, France, June 20-24, 1995), 1996, 139-143.

[8] A. V. Kisselev, V. A. Petrov, Eur. Phys. J. C, 36:1 (2004), 103-111, arXiv: hep-ph/0311356; 37:2 (2004), 241-249, arXiv: hep-th/0406062.

[9] S. K. Lucas, J. Comput. Appl. Math., 64:3 (1995), 269-282.

[10] J. Van Deun, R. Cools, Comp. Phys. Commun., 178:8 (2008), 578-590.

[11] J. T. Conway, Mon. Not. R. Astron. Soc., 316:3 (2000), 540-554.

[12] R. Mehrem, J. T. Londergan, M.H. Macfarlane, J. Phys. A: Math. Gen., 24:7 (1991), $1435-1453$.

[13] A. V. Kisselev, The Open Astronomy J., 2:1 (2009), 12-15.

[14] J. D. Jackson, Classical Electrodynamics, 3rd ed., John Wiley and Sons, New York, 1998.

[15] И. С. Градштейн, И.М. Рыжик, Таблицы интегралов, сумм, рядов и произведений, Физматгиз, М., 1963. 
[16] Г.Н. Ватсон, Теория бесселевых функиий, ИЛ, М., 1949.

[17] И. М. Гельфанд, Г. Е. Шилов, Обобщеннъе функиии, т. 1: Обобщеннъе функции и действия над ними, Физматгиз, М., 1959.

[18] А. П. Прудников, Ю. А. Брычков, О. И. Маричев, Интегралы и ряды, т. 2: Специальные функиии, Наука, М., 1983.

[19] Г. Бейтмен, А. Эрдейи, Высшие трансцендентные функции, т. 3: Эллиптические и автоморфные функции, функции Ламе и Матъе, Наука, М., 1967.

[20] Г. Бейтмен, А. Эрдейи, Высшие трансцендентные функции, т. 2: Функции Бесселя, функиии параболического ицлиндра, ортогональные многочлены, Наука, М., 1974.

Поступила в редакцию 6.11.2015, после доработки 26.12.2015 